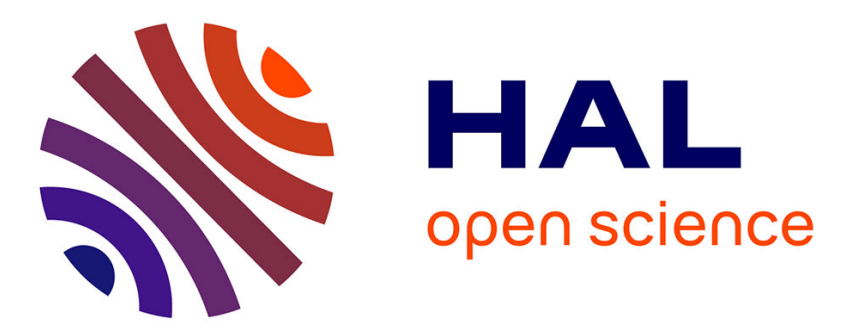

\title{
Influence of Co Content on the High-Temperature Oxidation Performance of (Ni,Co)-25Cr-0.4C-6Ta Cast
} Alloys

Patrice Berthod, Zohra Himeur

\section{To cite this version:}

Patrice Berthod, Zohra Himeur. Influence of Co Content on the High-Temperature Oxidation Performance of (Ni,Co)-25Cr-0.4C-6Ta Cast Alloys. Oxidation of Metals, 2018, 90 (1-2), pp.187-202. 10.1007/s11085-018-9833-x . hal-02187801

\section{HAL Id: hal-02187801 \\ https://hal.science/hal-02187801}

Submitted on 18 Jul 2019

HAL is a multi-disciplinary open access archive for the deposit and dissemination of scientific research documents, whether they are published or not. The documents may come from teaching and research institutions in France or abroad, or from public or private research centers.
L'archive ouverte pluridisciplinaire HAL, est destinée au dépôt et à la diffusion de documents scientifiques de niveau recherche, publiés ou non, émanant des établissements d'enseignement et de recherche français ou étrangers, des laboratoires publics ou privés. 


\title{
Influence of Co content on the high-temperature oxidation performance of $(\mathrm{Ni}, \mathrm{Co})-25 \mathrm{Cr}-0.4 \mathrm{C}-6 \mathrm{Ta}$ cast alloys
}

\author{
Patrice Berthod ${ }^{1,2}$, Zohra Himeur ${ }^{2}$ \\ ${ }^{1}$ Institut Jean Lamour (UMR CNRS 7198), Department CP2S, ${ }^{2}$ Faculty of Science and Technologies, University of \\ Lorraine, Postal Box 70239, 54506 Vandoeuvre-lès-Nancy, FRANCE \\ patrice.berthod@centraliens-lille.org,zohra.himeur5@etu.univ-lorraine.fr
}

\begin{abstract}
Tantalum carbides $(\mathrm{TaC})$ are classical strengthening particles, like chromium carbides, in equiaxed cast Cr-rich cobalt-based alloys. When they are of a eutectic nature and in quantity high enough, they may be of great interest for applications at elevated temperature, due to their favorable script-like morphology and their high stability at high temperature. Unfortunately they cannot be so easily obtained in Cr-rich nickel-based alloys of similar composition. This can be resolved by adding cobalt in substitution to nickel but such chemical modification may change the high temperature oxidation behavior in the wrong direction. In this work several alloys, all containing $25 \% \mathrm{Cr}, 0.4 \% \mathrm{C}$ and $6 \% \mathrm{Ta}$ (wt.\%), were elaborated and studied at a high temperature: $1127^{\circ} \mathrm{C}$. It appears that the $27 \mathrm{wt} . \%$ Co content is high enough to allow obtaining primary carbides which are essentially TaC. The alloys rich enough in Co containing only tantalum carbides are clearly disadvantaged in term of oxidation resistance at high temperature by the high Co content and by the absence of interdendritic chromium carbides known to act as useful $\mathrm{Cr}$ reservoirs. Among the six studied alloys these are the three ones for which $\mathrm{Co} / \mathrm{Ni}>1$ that demonstrated starts of catastrophic oxidation before 24 hours of exposure to air.
\end{abstract}

Keywords: Nickel-based alloys; Cobalt-based alloys; Chromium carbides; Tantalum carbides; High temperature oxidation; Thermodynamic calculations

Post-print version of the article Oxid Met (2018) 90:187-202; https://doi.org/10.1007/s11085-018-9833-x

\section{INTRODUCTION}

Primary carbides belong to the earliest metallurgical means employed for strengthening the cast metallic alloys used at high temperature under mechanical stresses [1,2]. Beside chromium carbides, tantalum carbides can play a special role, for example in the MarM-509 alloy [3-5]. Even if many MC carbides may be obtained in conventionally cast Ni-based [6] or Co-based [7] alloys, TaC carbides keep an interest high enough to be still employed in numerous superalloys [8]. Primary carbides are principally used in equi-axed polycrystalline alloys and these ones often contain also high contents in chromium to resist oxidative chemical aggression at high temperature [9]. Chromium is a potential competitor for tantalum in the formation of carbides. Tantalum is not necessarily a carbide-former stronger than chromium and, in some cases, chromium carbides may precipitate preferentially to TaC. For example, during the solidification of chromium-rich cobalt-based [10] and iron-based [11] alloys, TaC forms at the expense of $\mathrm{Cr}_{7} \mathrm{C}_{3}$ or $\mathrm{Cr}_{23} \mathrm{C}_{6}$. It is the contrary for Cr-rich nickel-based alloys [12]. Cobalt and nickel are often associated to one another in commercial alloys. For example Co can be present in nickel alloys for solid solution strengthening purpose and $\mathrm{Ni}$ can be added to cobalt alloys to extend the temperature range of thermodynamic stability of the high temperature austenitic face centered cubic crystalline structure of cobalt. Other possible types of association of nickel and cobalt aim to the better high temperature oxidation behavior brought by nickel to a cobalt matrix by expediting chromium diffusion towards the oxidation front, or to the stabilization of the TaC carbides induced by cobalt addition to a nickel matrix [13]. 
The aim of the present work is to explore how the behavior in oxidation at high temperature of carbidescontaining nickel-chromium cast alloys evolves when more and more cobalt is added to stabilize tantalum carbides more and more efficiently.

\section{EXPERIMENTAL}

To investigate how the behavior in oxidation at high temperature of cast equi-axed nickel-based alloys, rich in chromium, strengthened by carbides and containing a high tantalum content, may evolve when nickel is increasingly substituted by cobalt, six alloys of the Ni-Co-25Cr-0.4C-6Ta were elaborated. Their targeted chemical compositions are displayed in Table 1. These alloys were synthesized by classical foundry, by melting together parts of pure elements (Ni, Co, Cr and Ta from Alfa Aesar, and graphite, purity higher than $99.9 \%$ in weight). This was achieved in the water cooled copper crucible of a CELES high frequency induction furnace $(50 \mathrm{~kW}$, about $100 \mathrm{~Hz})$ in which the molten alloys also solidified. All the alloys were kept in the obtained as-cast state, without any postsolidification heat treatment, in order to stay in the usual conditions of use of many of the commercial alloys (e.g. Mar-M322 or 509 of which the present alloys can be considered the simplified versions $[1,2,8]$.

The six obtained ingots, all weighing about 40 grams, were each cut in four parts. One of them was kept for preparing the sample devoted to the as-cast microstructures control and a second one was specially prepared for the oxidation tests at high temperature. The chosen temperature and duration were $1127^{\circ} \mathrm{C}(1400 \mathrm{~K})$ and 24 hours (a day). The samples were placed in a muffle furnace (Nabertherm) and exposed to laboratory air at the targeted temperature.

The obtained oxidized samples were first covered by an extra-thin gold coating. They were then immersed in a $50^{\circ} \mathrm{C}$-heated Watt's bath. They staid located between two nickel coupons which played the role of anodes. The samples were polarized at a high potential allowing a constant electrical current corresponding to the $1.6 \mathrm{~A} \times \mathrm{dm}^{-2}$ current density known to allow a good conditions of coating. After 2 hours of electrolytic coating, the samples were surrounded by metallic nickel shell thick enough to thereafter protect the external scale during the cutting necessary to the preparation of cross-sections. Cutting of each Ni-coated oxidized sample in two halves was carried out using a metallographic Buehler Delta Abrasimet 5000 metallographic saw. The obtained halves were embedded in a cold resin mixture (ESCIL). These cross-sectional samples prepared from the oxidized alloys and the samples for as-cast microstructure observation were polished using SiC papers from 240-grit to 1200-grit. They were then washed and ultrasonically cleaned to remove all possible attached SiC particles. Final polishing, carried out using textile supporting very fine $(1 \mu \mathrm{m})$ hard particles, led to mirror-like states.

Metallographic examinations were done using a scanning electron microscope (SEM, JEOL, JSM-6010LA model) equipped with an Energy Dispersion Spectrometer (EDS). The most used mode for imaging was Back Scattered Electrons one (BSE). The applied voltage was $20 \mathrm{kV}$. The EDS full frame analyses were carried out at $\times 250$ on three locations (random positioning) to control the general chemical compositions of the alloys. Spot analyses were performed to identify the different phases present in the alloys, and the different oxides seen in the external scales and the internal oxides appeared in the subsurface.

Some thermodynamic calculations were also carried out. For that one used the Thermo-Calc software (version $\mathrm{N}$ ) [14] and a database. This database was the SSOL database [15] which was enriched with additional binary and ternary systems involving Ta, element which was missing in the original database. This enriched version which was then used contained the descriptions of the Ta-Ni, Ta-Co, Ta-Cr and Ta-C binaries, and of the Co-Ta-C, Ni-Ta-C, Cr-Ta-C ternaries.

\section{RESULTS}

\section{Chemical compositions and microstructures of the alloys in their as-cast conditions}

As this can be verified by the EDS analysis results presented in Table 2, the obtained chemical compositions well meet the targeted ones. However one must note that the $\mathrm{Cr}$ contents are a little higher than what it was wished. This is also true for the Ta contents which are greater than expected. This phenomenon is usual when full frame EDS is used for microstructures in which a part of tantalum is involved in coarse TaC carbides. Nevertheless one can consider that the Ta contents in alloys are themselves close to the wished ones. EDS does not operate for carbon since this one is a light element and, in addition, present in very low quantity (wished contents of only $0.4 \%$ wt. $\%$ ). This is by considering the densities of the obtained carbide network which allows estimate that the $0.4 \mathrm{wt} . \% \mathrm{C}$ is well 
respected in all alloys. Periodically performed spark spectrometry analysis of alloys of this family, elaborated with the same protocol, constantly confirms that the targeted carbon contents are always obtained without any loss.

The rather rapid solidification and solid state cooling induced crystallization and subsequent solid state transformations which did not really respect the phase diagram (Figure 1). Indeed, it was expected that the NiCo0 and NiCo1 alloys will contain no TaC but only chromium carbides. For the NiCo2 and NiCo3 alloys it was expected that they contain both chromium carbides and $\mathrm{TaC}$. To finish the $\mathrm{NiCo} 4$ and $\mathrm{NiCo} 5$ alloys were expected to contain only TaC. In fact, it appears that the as-cast microstructures of the two Ni-richest alloys (NiCo0 and NiCo1: Figure 2) contain significant parts of tantalum carbides (white particles when observed with a SEM in BSE mode), beside the chromium carbides (dark particles) which are notwithstanding more present than the TaC. Tantalum carbides seem to be more present than chromium carbides in the NiCo2 alloy (Figure 3) although they ought to remain the minor part of the carbide population, the chromium carbides being expected to be the major part. The NiCo3 alloy contains almost only tantalum carbides. In this alloy the chromium carbides are present only as rare small particles (Figure 3). The NiCo4 and NiCo5 alloys (Figure 4) present microstructures which do not contain chromium carbides, and their carbide network is then only constituted of tantalum carbides. Concerning the new phases which ought to appear during cooling below about $1000^{\circ} \mathrm{C}$ according to the phase diagram (e.g. the $\mathrm{Ni}_{3} \mathrm{Ta}_{\mathrm{T}}$ ntermetallic compound), they were not observed. These small disagreements with what was predicted by thermodynamic calculations can be easily explained by the rapid cooling during both solidification (e.g. segregation of Ta rejected in the liquid at the solid/liquid interface) and solid state cooling (no time for nucleation and growth of the new phases).

\section{Oxidation products and subsurface modifications: qualitative observations}

During the twenty-four hours spent at $1127^{\circ} \mathrm{C}$, all alloys suffered from oxidation by the laboratory air. In all cases a continuous oxide scale developed all around the samples. Internal oxidation also happened and an outer zone of alloy subsurface lost the carbides which were initially present there. Impoverishments in chromium and in tantalum occurred in subsurface These surface and subsurface modifications are illustrated by SEM/BSE micrographs taken on the prepared cross-sections: in Figure 5 for the two Ni-richest alloys (NiCo0 and NiCo1), in Figure 6 for the two intermediate alloys ( $\mathrm{NiCo} 2$ and NiCo3) and in Figure 7 for the two Co-richest alloys (NiCo4 and $\mathrm{NiCo5}$ ). In all these figures the upper micrograph gives a general view of the oxidized surface and of the deteriorated subsurface. These zones are more detailed by the higher magnification micrograph displayed bellow.

Two types of oxides formed for the three first alloys ( $\mathrm{NiCo} 0, \mathrm{NiCo} 1$ and $\mathrm{NiCo} 2)$ which are richer in nickel than in cobalt. These oxides are first chromia $\left(\mathrm{Cr}_{2} \mathrm{O}_{3}\right)$, which represents the greatest part and which is exclusively present in the external scale, and second a complex oxide which involves chromium and tantalum simultaneously $\left(\mathrm{CrTaO}_{4}\right)$. The greatest part of this second oxide is present in the subsurface, principally in the outer half of the carbide-free zone, close to the alloy/external scale interface. In addition, this complex oxide is also present as a wavy oxide film immersed in the chromia external scale.

The same oxides, chromia and the complex oxide of chromium and are also present in the three last alloys, $\mathrm{NiCo}$, NiCo4 and $\mathrm{NiCo5}$, which are richer in cobalt than in nickel. As for the former alloys, chromia is present, again as an external scale, but another oxide, of a MO type is also present. This one involves of Ni and Co together when $\mathrm{Ni}$ s still present in rather high quantity (case of NiCo3). In the case of the two Co-richest alloys, it involves only Co. This MO oxide is present too as an external oxide separating chromia and air. These (Co,Ni)O and $\mathrm{CoO}$ oxides, visible thanks to their tint paler than the chromia's one, are known to be not so protective as $\mathrm{Cr}_{2} \mathrm{O}_{3}$. A third oxide can also be found beside chromia and the $\mathrm{MO}$ oxides: the spinel $\mathrm{CoCr}_{2} \mathrm{O}_{4}$. It is mainly present at the chromia/CoO interface.

\section{Oxidation products and subsurface modifications: quantitative results}

Even if it was obvious that oxide spallation occurred during the cooling after the end of isothermal oxidation, it was possible to assess the thickness of the external oxide scale. This one was measured in ten distinct locations, representative of the general state of the oxidized surface of each sample. The average and standard deviations are given in the second column of Table 3 (the first results column). One can see that the average oxide thickness globally tends to increase when the cobalt content increases. In fact, one can consider that the external scales developed over the three nickel-richest alloys are of the same thickness. This one is rather low: 5 to $6 \mu \mathrm{m}$. The three other alloys indisputably constitute another group, which is characterized: first by a much thicker external oxide 
scales (average thickness equal to 3 or 5 times the ones obtained for the three first alloys), and second, regard to the much by a worse thickness homogeneity as revealed by the high values of standard deviation.

The depth over which alloys had lost their carbides is obviously also strongly dependent on the cobalt content (Table 3, third column, e.g. second results column). Rather high for the nickel-based NiCo0 alloy, the average value (issued from ten values too) decreases of one third, from 30 to $20 \mu \mathrm{m}$ globally. From the NiCo2 alloy to the NiCo3 one, the depth of carbides disappearance is suddenly doubled (typical $40 \mu \mathrm{m}$ ). By enrichment in Co, the depth of carbide-free zone decreases again, to reach almost the carbide-free depth of the NiCo0 alloy. One must also note that the values of standard deviation are higher.

The local changes in the alloy in chemical composition due to oxidation can be illustrated by the concentrations profiles acquired from the external oxide scale / alloy interface. They are presented in Figure 8 for the major elements, and in Figure 9 for the minor elements, only three of the six alloys. Each of them represents one of the three kinds of alloys: the Ni-richest ones, the medium ones, and the Co-richest ones. The profiles of Cr depletion (Figure 8) are often constituted of an outer part characterized by a stationary Cr content followed by a rather high gradient. This outer part corresponds more or less to the carbide-free zone in the case of the two Ni-richest alloys. Deeper there is a second gradient, which starts from the frontier between the carbide-free zone and the inner part of alloy in which carbides are still present. This second gradient is low in the case of the Ni-richest alloys. For the Corichest alloys the second $\mathrm{Cr}$ gradient is more marked and it becomes low or inexistent only much deeper, where the alloy not affected by oxidation starts. When observing these Cr-concentration profiles one can see that the chromium content in extreme surface of the sample, i.e. very close to the scale/alloy interface, tends decreasing when the global cobalt content in alloy increases. Up to ten EDS spot analyses were carried out in the alloy in extreme surface, all along the interface with the external scale. The average and standard deviation values were calculated for the obtained $\mathrm{Cr}$ contents. These ones are presented in Table 4. The average chromium content in extreme surface obviously decreases when the cobalt content in the alloy increases. This confirms the tendency shown by the concentration profiles. One can also say that there are to typical levels, $16-17 \mathrm{wt} \% \mathrm{Cr}$ for the three Ni-richest alloys and $12-13 \mathrm{wt} . \% \mathrm{Cr}$ for the three Co-richest ones.

\section{DISCUSSION}

The studied alloys belong to the same family as the alloys recently studied by Z. Himeur [13]. As for these later ones, the as-cast microstructures of the present alloys do not respect exactly the microstructures predicted by Thermo-Calc. This is not surprising. Indeed solidification and solid state cooling were fast, consequence of the use of a water-cooled copper crucible. This notably led to the rejection of the heavy tantalum atoms in the liquid close to the solidification front, with consequently the appearance of interdendritic zone rich in segregated Ta. The subsequent time spent at high temperature between solidification and the end of cooling was not long enough to chemically homogenize the matrix. The chemical heterogeneity at the microstructure size scale inherited from this segregation phenomenon can be a first possible reason of the appearance of tantalum carbides in the cobalt-free NiCo0 alloy despite Thermo-Calc did not predict their presence. However, one can consider the bottom halves of the $\times 250 \mathrm{SEM} / \mathrm{BSE}$ micrographs presented in Figures 5-7, for having a general view of the cross-sections of the oxidized samples. In these zones far from surface, and therefore where the microstructure is too deep for having been affected by oxidation, it appears that a significant part of the TaC appeared during solidification staid stable during the rather long exposure at $1127^{\circ} \mathrm{C}$. Furthermore, the $1127^{\circ} \mathrm{C}$-stabilized microstructure of the NiCo1 alloy (bottom part of the corresponding $\times 250$ micrograph (Fig. 5, top left) only contains $\mathrm{TaC}$ as carbide phase. This was not expected since thermodynamic calculations predicted the presence of chromium carbides only, with eventually very little quantity of $\mathrm{TaC}$. The $\mathrm{NiCo} 2$ and $\mathrm{NiCo} 3$ alloys, which ought to contain significant quantities of chromium carbides and of tantalum carbides, contained only few chromium carbides and essentially tantalum carbides. After exposure at $1127^{\circ} \mathrm{C}$ for 24 hours their carbide population was composed of TaC exclusively (Figure 6, bottom micrographs, bottom halves). Concerning the two last alloys, NiCo4 and NiCo5, Thermo-Calc calculations led to the presence of only $\mathrm{TaC}$. This was effectively verified experimentally, in the as-cast state as well as in the part of subsurface not affected by oxidation.. To finish one can note that the surface fractions of TaC in the as-cast microstructures seem qualitatively similar to one another between the four cobalt-richest alloys (free or almost free of chromium carbides) and between all alloys excepted $\mathrm{NiCo} 0$ concerning the microstructures stabilized at $1127^{\circ} \mathrm{C}$ (carbides exclusively $\mathrm{TaC}$ ). 
To summarize, these observations, which are often in disagreement with the thermodynamic calculations, demonstrate that the used database obviously needs to be improved to deliver more correct results in presence of Ta. Consequently one must use only the experimental results, which lead to consider that:

- the difference in high temperature oxidation behavior between the $\mathrm{NiCo} 0$ and $\mathrm{NiCo} 1$ alloy can be due to the difference in nature of the carbide population as well as to the presence of $14 \mathrm{wt} . \% \mathrm{Co}$

- the differences in oxidation behavior between the other alloys should be exclusively attributed to the Co content in the matrix.

The oxidation runs were carried out at a rather elevated temperature, of the same level as for the solutioning treatment usually applied during one or two hours to the commercial precipitation-hardened superalloys, prior to the precipitation treatment. Therefore the present alloys maybe considered as already chemical homogenized for the major part of the oxidation process. After cross-sectional metallographic preparation one observed and characterized the oxidation products which formed and the microstructure consequences of oxidation in subsurface. It was found that the oxide scales formed on the $\mathrm{NiCo} 0$ alloy and the NiCo1 alloy were of the same constitution and of the same average thickness. The carbide-free zone was significantly deeper in the NiCo0 alloy than in the NiCo1 alloy. The latter observation may be interpreted as follows: during the whole oxidation, the chromium carbides existing permanently in the NiCo0 alloy dissolved over an increasing depth from the scale/alloy interface; this phenomenon is due to the local decrease in $\mathrm{Cr}$ in the neighbor matrix (Figure 8) subsequently to the $\mathrm{Cr}$ diffusion toward the oxidation front. For the same reason, but this time concerning tantalum (Figure 9), the neighbor tantalum carbides dissolved and the Ta atoms well diffused along the interdendritic spaces free of chromium carbides. This effectively may lead to a rather deep carbide-free zone and to the presence of many $\mathrm{CrTaO}_{4}$ oxides in the outer part of this carbide-free zone. What occurred in the NiCo1 alloy was probably different: the tantalum carbides, more present than in the $\mathrm{NiCo} 0$ alloy, had a little grown by profiting from the carbon atoms released by the disappearing chromium carbides, and they were present in subsurface for a longer time. Indeed, the diffusion of the Ta atoms is not as easy as the chromium atoms and this resulted in less deep carbide-free zone forthe same duration at $1127^{\circ} \mathrm{C}$.

The NiCo2 alloy globally behaved as the NiCo1 one, with as results sensibly the same average scale thickness and the same carbide-free depth. However one can note a decrease of about $1 \mathrm{wt} . \%$ of the average $\mathrm{Cr}$ content in extreme surface. This possibly means that the volume diffusion of $\mathrm{Cr}$ in the matrix may be not so easy as for the two former alloys. This is a possible effect of an important presence of cobalt ( $27 \mathrm{wt} \% \mathrm{Co})$ substituting nickel in the matrix.

By enriching more the alloy with cobalt, drastic changes occurred. With about $41 \mathrm{wt}$ \% Co against $27 \mathrm{wt} . \% \mathrm{Ni}$, the next alloy, NiCo3, is no longer nickel-based but cobalt-based. This change in base is obviously accompanied by a sudden change in behavior. Probable consequence of less easy volume $\mathrm{Cr}$ diffusion, this alloy lost its total chromia-forming behavior before reaching the end of the 24 hours stage. Consequently other oxides formed in addition to the $\mathrm{Cr}_{2} \mathrm{O}_{3}$ and $\mathrm{CrTaO}_{4}$ which were the only ones to form in the same conditions for the three Ni-richer alloys. Indeed (Co,Ni)O was observed. This loss of the chromia-forming behavior is due to the decrease in $\mathrm{Cr}$ content in extreme surface of about $3 \mathrm{wt} . \% \mathrm{Cr}$ more. Less slowed down by multi-constituted oxide scale less protective than chromia, oxidation was faster. This had as result a migration of $\mathrm{Cr}$ and $\mathrm{Ta}$ toward the oxidation front, and consequently a more extended carbide-free zone. The NiCo4 and NiCo5 alloys behaved worse than the NiCo3 alloy. In their case, the difficult $\mathrm{Cr}$ diffusion induced a more dangerous exposure of the outer parts of alloys to oxidation. This led to a generalized oxidation of Co together with chromium. Greater parts of CoO and of spinel $\mathrm{CoCr}_{2} \mathrm{O}_{4}$ oxides were obtained, and an inward penetration of oxidation started, as revealed by the irregular alloy/oxides frontier.

\section{CONCLUSION}

So, the increase in cobalt content induced a stabilization of the $\mathrm{TaC}$ carbides. Unfortunately, at the same time this hindered more and more Cr migration toward the oxidation front. Both grain boundary / interdendritic diffusion were impacted by the absence of the interdendritic chromium carbides and by the less easy diffusion in volume through the matrix. The two moderately Co-added alloys, NiCo1 and $\mathrm{NiCo} 2$, may present interesting mechanical properties at high temperature since they contain a strengthening network of interdendritic tantalum carbides, despite their still rather intrinsically weak Ni-rich matrix (compared to a cobalt-based one), without conceding the good high temperature oxidation behavior of a real chromium-rich Ni-based alloy. The high temperature oxidation resistance of the three other alloys, which contain more cobalt than nickel, must be improved to allow exploiting the 
reinforcement by $\mathrm{TaC}$. This can be done globally by adding several wt.\% Cr points, either in the whole alloy (enriching in $\mathrm{Cr}$ its chemical composition) or only in the subsurface (by depositing chromium to diffuse over a limited depth, as allowed by some Chemical Vapor Deposition techniques). Although that the available database was obviously not efficient enough to correctly describe the metallurgical states of these quinary alloys, thermodynamic calculations in the cobalt-rich half part of the isopleth section (Figure 10) would show qualitatively that some microstructural consequences, either globally (Cr-enriched alloy) or locally (Cr-enriched subsurface) ought to be taken into account for their global high temperature properties.

\section{References}

1. C. T. Sims, W. C. Hagel, The Superalloys, John Wiley and Sons, 1972.

2. E. F. Bradley, Superalloys: A technical guide, Metals Park: ASM International, 1988.

3. H. Morrow, W. P. Danesi, D. L. Sponseller, Cobalt, 4, 93 (1973).

4. R. L. Ammon, L. R. Eisenstatt, G. O. Yatsko, Journal of Engineering for Power, 103(2), 331 (1981).

5. J. Reuchet, L. Remy, Metallurgical Transactions A: Physical Metallurgy and Materials Science, 14(1), 141 (1983).

6. P. Berthod, Advanced Materials Letters, 8(8), 866 (2017).

7. P. Berthod, Advances in Materials Science and Engineering, article ID 4145369, https://doi.org/10.1155/2017/4145369 (2017).

8. M. J. Donachie, S. J. Donachie, Superalloys: A Technical Guide ( $2^{\text {nd }}$ Edition), Materials Park: ASM International, 2002.

9. D. Young, High Temperature Oxidation and Corrosion of Metals, Amsterdam: Elsevier Corrosion Series, 2008.

10. P. Berthod, S. Michon, L. Aranda, S. Mathieu, J. C. Gachon, Calphad, 27, 353 (2003).

11. P. Berthod, Y. Hamini, L. Aranda, L. Héricher, Calphad, 31, 351 (2007).

12. P. Berthod, L. Aranda, C. Vébert, S. Michon, Calphad, 28, 159 (2004).

13. Z. Himeur, report of Master trainee period, University of Lorraine (Nancy, France), 2017.

14. Thermo-Calc version N: "Foundation for Computational Thermodynamics" Stockholm, Sweden, Copyright (1993, 2000). www.thermocalc.com

15. SSOL database, SGTE Solutions Database, Scientific Group Thermodata Europe, Bo Sundman, Stockholm, Sweden. 
TABLE 1. Targeted compositions for the six alloys of the study (in wt.\%).

\begin{tabular}{|c|c|c|c|c|c|}
\hline Alloys & $\mathrm{Ni}$ & $\mathrm{Co}$ & $\mathrm{Cr}$ & $\mathrm{Ta}$ & $\mathrm{C}$ \\
\hline "NiCo0" & Bal. & 0 & 25 & 6 & 0.4 \\
\hline "NiCo1" & Bal. & 13.7 & 25 & 6 & 0.4 \\
\hline "NiCo2" & Bal. & 27.4 & 25 & 6 & 0.4 \\
\hline "NiCo3" & 27.4 & Bal. & 25 & 6 & 0.4 \\
\hline "NiCo4" & 13.7 & Bal. & 25 & 6 & 0.4 \\
\hline "NiCo5" & 0 & Bal. & 25 & 6 & 0.4 \\
\hline
\end{tabular}

TABLE 2. Obtained chemical compositions (in wt.\%); average and standard deviation of three full-frame SEM/EDS measurements carried out at the $\times 250$ magnification)

\begin{tabular}{|c|c|c|c|c|c|}
\hline Alloys & $\mathrm{Ni}$ & $\mathrm{Co}$ & $\mathrm{Cr}$ & $\mathrm{Ta}$ & $\mathrm{C}$ \\
\hline "NiCo0" & Bal. & $/$ & $25.5 \pm 0.1$ & $6.5 \pm 0.2$ & "0.4"* \\
\hline "NiCo1" & Bal. & $14.3 \pm 0.2$ & $25.4 \pm 0.4$ & $5.6 \pm 0.9$ & “0.4"* \\
\hline "NiCo2" & Bal. & $27.2 \pm 0.2$ & $25.4 \pm 0.1$ & $6.5 \pm 0.2$ & "0.4"* \\
\hline "NiCo3" & $25.4 \pm 0.3$ & Bal. & $25.7 \pm 0.4$ & $6.9 \pm 0.2$ & "0.4"* \\
\hline "NiCo4" & $12.7 \pm 0.5$ & Bal. & $26.2 \pm 0.2$ & $6.6 \pm 0.8$ & "0.4"* \\
\hline "NiCo5" & $/$ & Bal. & $25.3 \pm 0.2$ & $7.2 \pm 0.5$ & "0.4"* \\
\hline
\end{tabular}

*: carbon contents not controlled by EDS but assumed to be well respected according to the practical knowledge for the elaboration protocol for many alloys before, and confirmed by the obtained density of carbides

TABLE 3. Average and standard deviations of the thickness of the external oxide scale (first results column) and of the carbide-free depth (second results column) for the six alloys after $24 \mathrm{~h}$-oxidation at $1127^{\circ} \mathrm{C}$

\begin{tabular}{|c|c|c|}
\hline Alloys & Oxide scale thickness $(\mu \mathrm{m})$ & Carbide-free zone depth $(\mu \mathrm{m})$ \\
\hline "NiCo0" & $5.9 \pm 1.6$ & $28.4 \pm 2.8$ \\
\hline "NiCo1" & $6.0 \pm 2.3$ & $18.1 \pm 4.8$ \\
\hline "NiCo2" & $4.9 \pm 0.7$ & $18.7 \pm 1.5$ \\
\hline "NiCo3" & $16.4 \pm 3.9^{*}$ & $41.9 \pm 7.2$ \\
\hline "NiCo4" & $24.4 \pm 4.8^{*}$ & $36.0 \pm 7.7$ \\
\hline "NiCo5" & $28.6 \pm 22.6^{* *}$ & $29.0 \pm 6$ \\
\hline
\end{tabular}

*,**: high or very high values of standard deviation due to very heterogeneous oxidation behavior all along the surface

TABLE 4. Average and standard deviation for the chromium content in alloy close to the external scale/alloy interface (three measurements)

\begin{tabular}{|c|c|}
\hline Alloys & Chromium content in extreme surface (wt.\%) \\
\hline "NiCo0" & $16.8 \pm 0.6$ \\
\hline "NiCo1" & $16.9 \pm 0.1$ \\
\hline "NiCo2" & $16.1 \pm 0.4$ \\
\hline "NiCo3" & $13.0 \pm 3.3$ \\
\hline "NiCo4" & $13.1 \pm 0.3$ \\
\hline "NiCo5" & $12.4 \pm 0.3$ \\
\hline
\end{tabular}




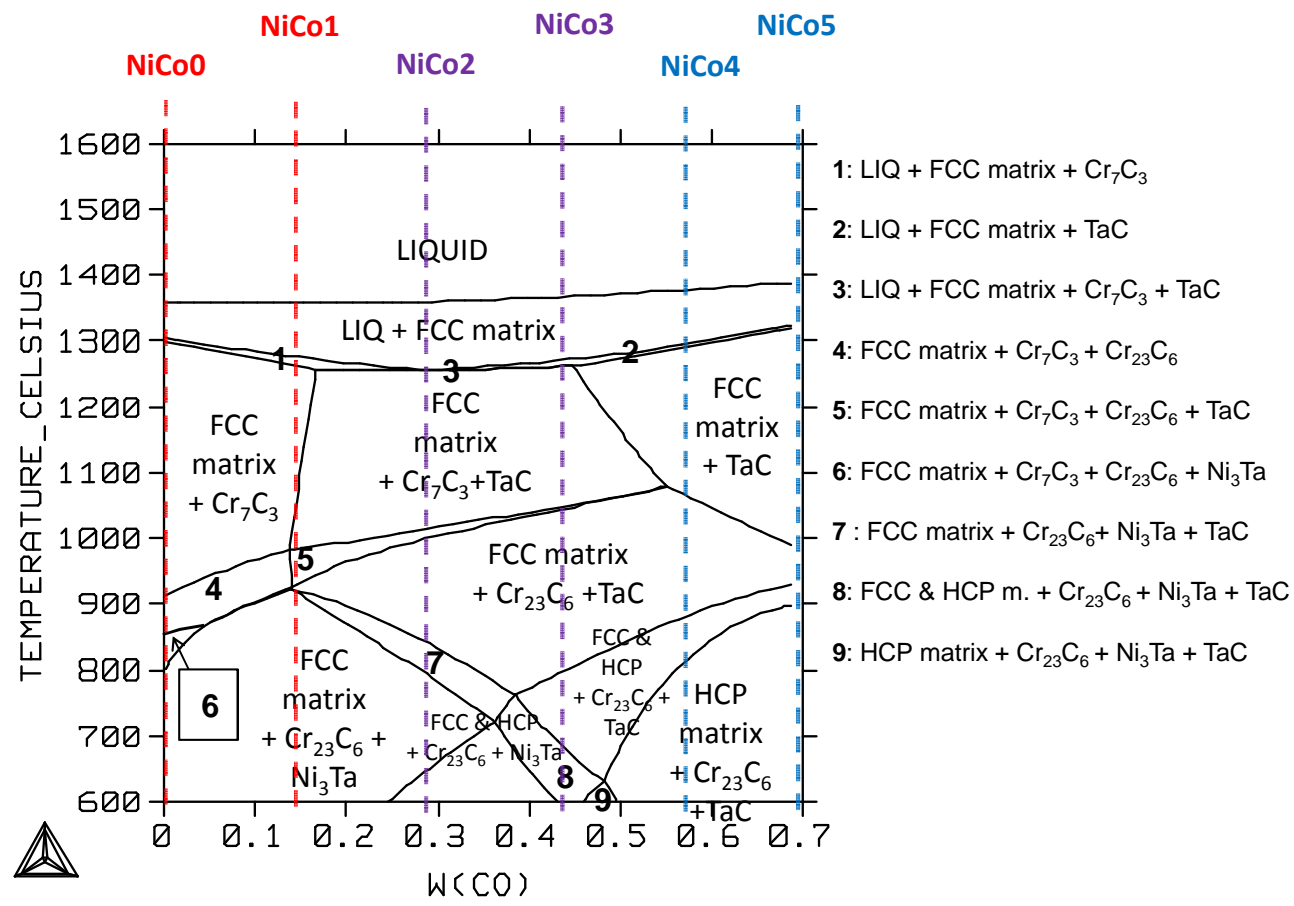

FIGURE 1. $\{25 \mathrm{Cr}-6 \mathrm{Ta}-0.4 \mathrm{C}\}$-isopleth section of the Ni-Co-Cr-C-Ta diagram computed by Thermo-Calc and position of the six studied alloys 


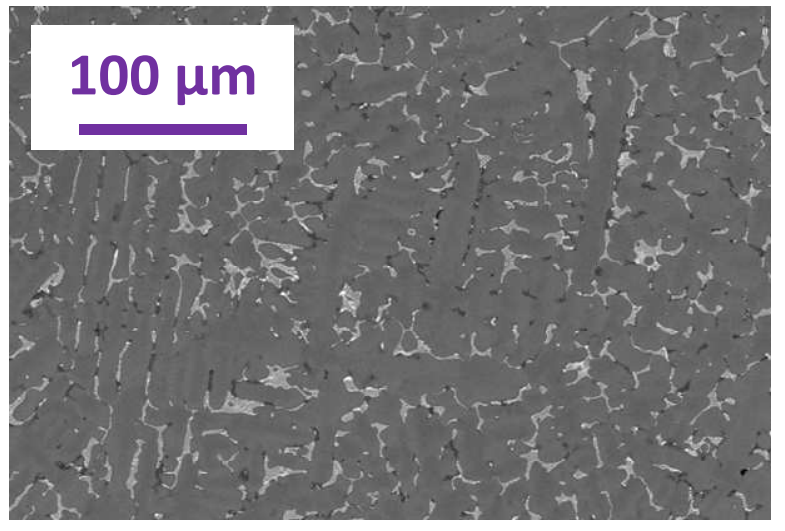

NiCo2 alloy (as-cast)
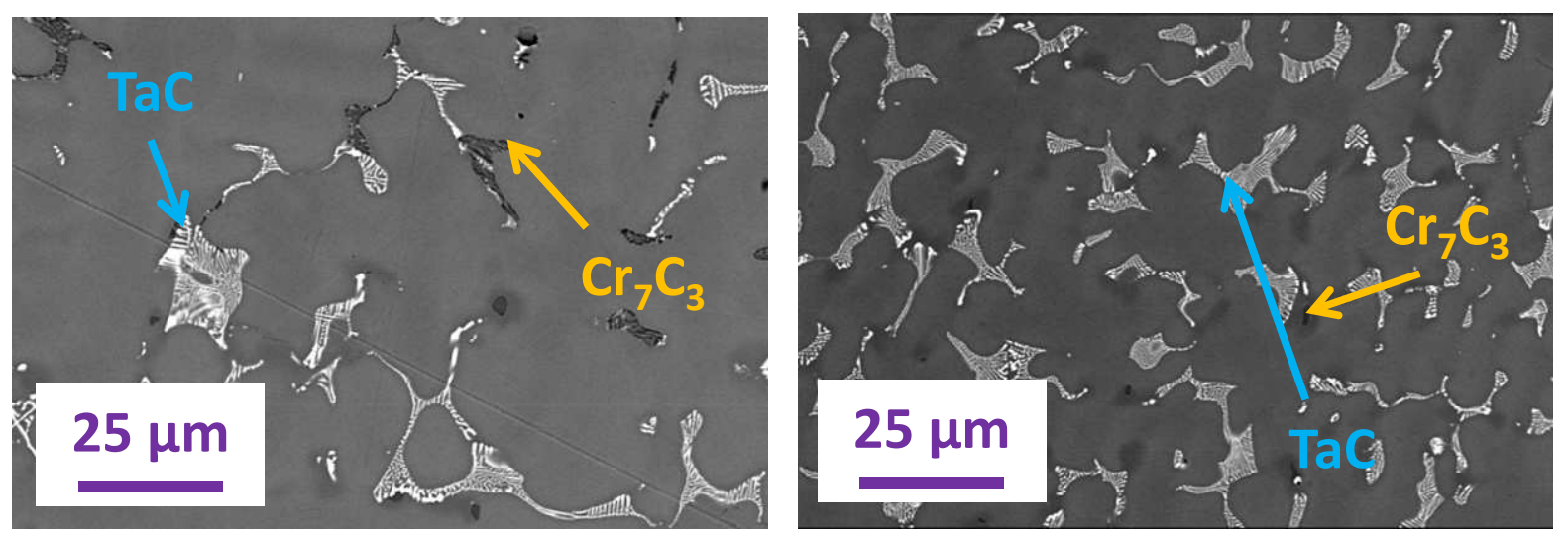

FIGURE 3. As-cast microstructures of the two medium alloys (SEM/BSE; top: $\times 250$; bottom: $\times 1000$ ) 

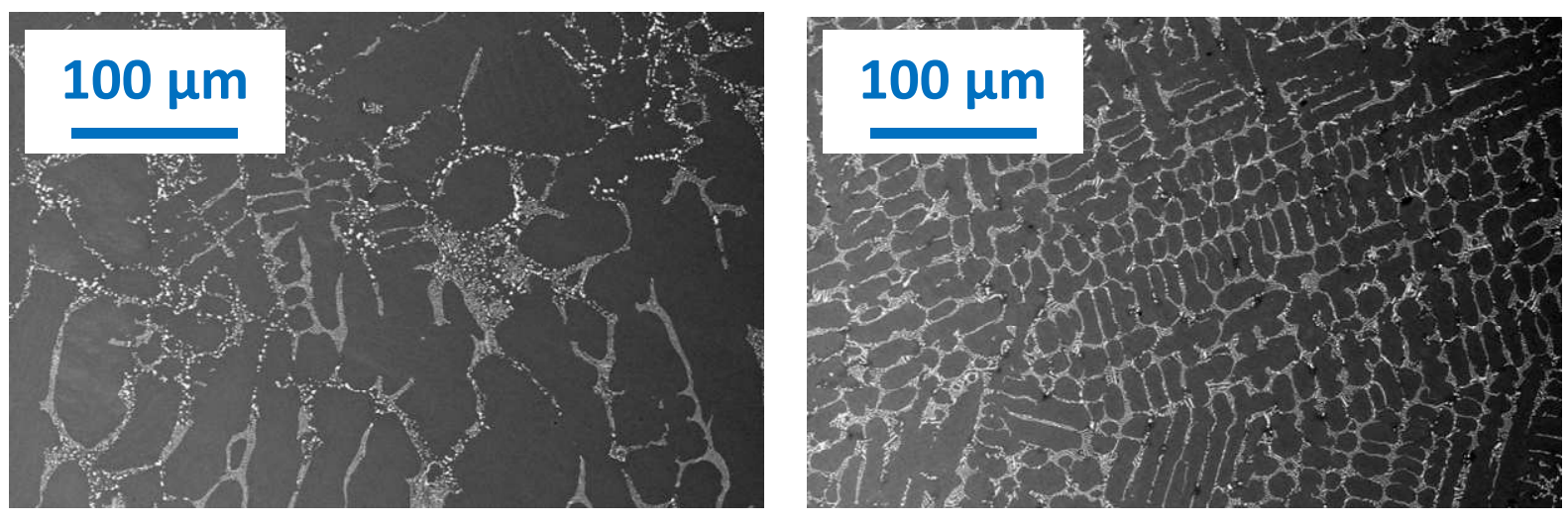

NiCo4 alloy (as-cast)

\section{NiCo5 alloy (as-cast)}
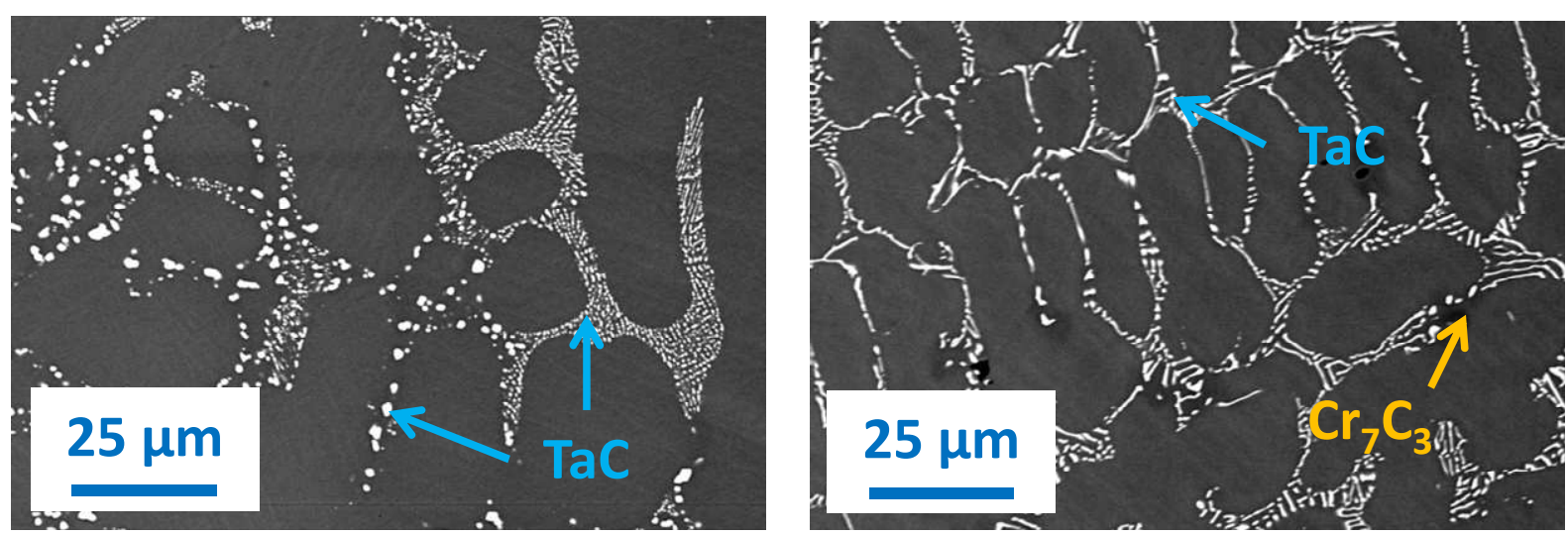

FIGURE 4. As-cast microstructures of the two Co-richest alloys (SEM/BSE; top: $\times 250$; bottom: $\times 1000$ ) 


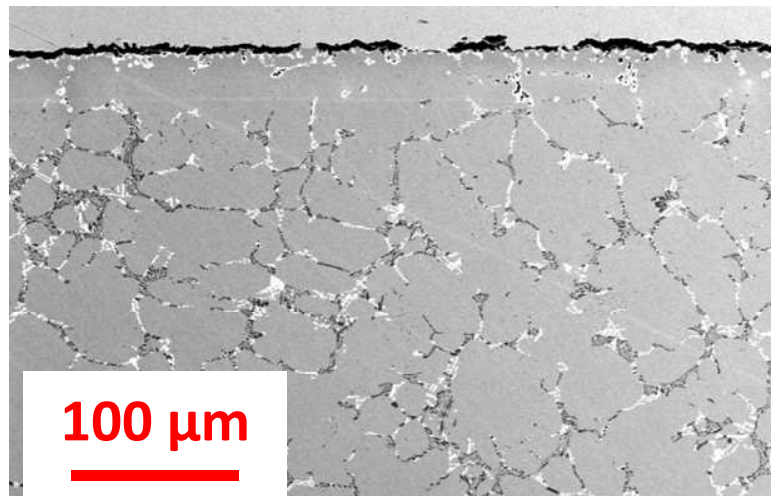

$\mathrm{NiCoO}$ alloy $\left(24 \mathrm{~h} 1127^{\circ} \mathrm{C}\right)$
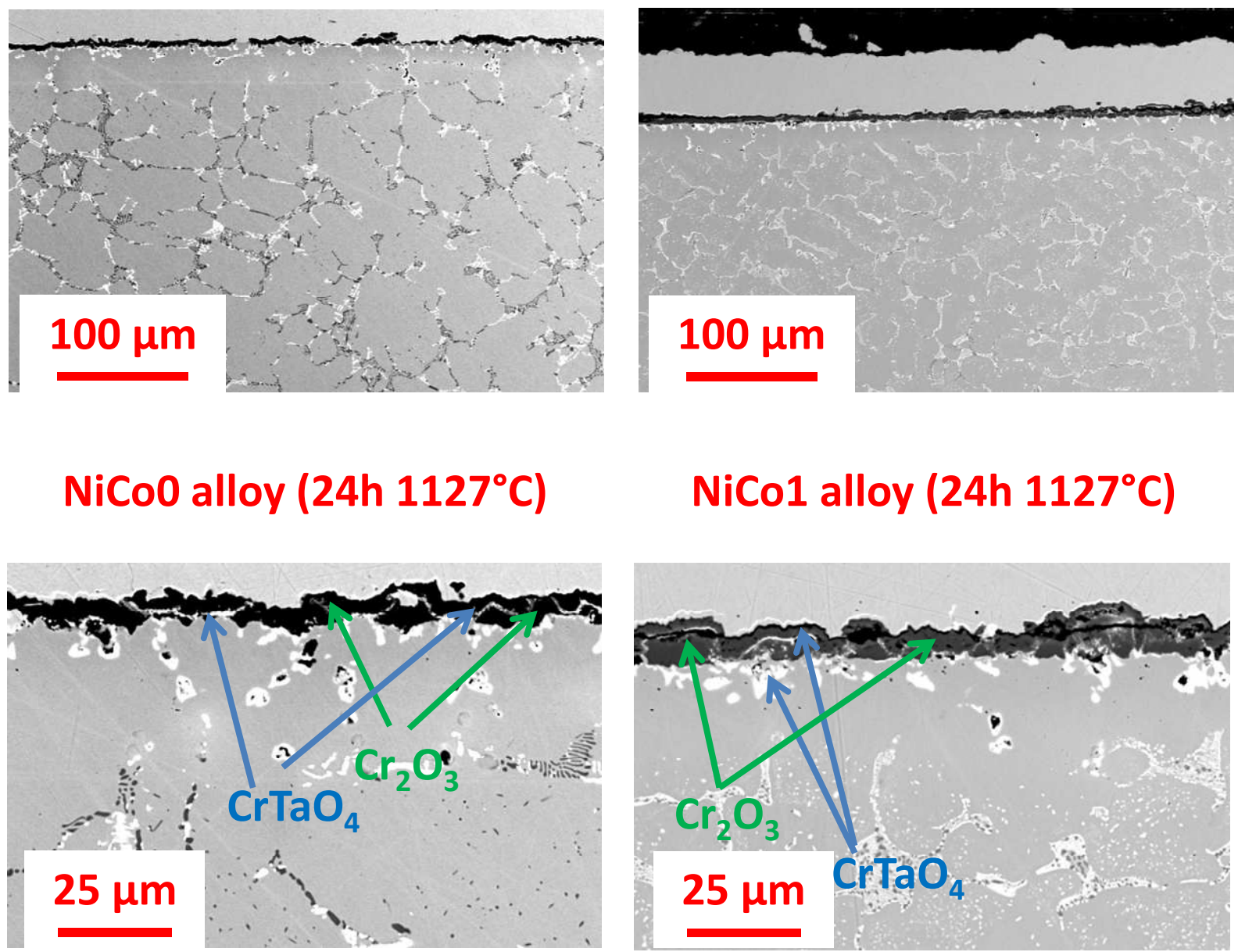

NiCo1 alloy $\left(24 \mathrm{~h} 1127^{\circ} \mathrm{C}\right)$

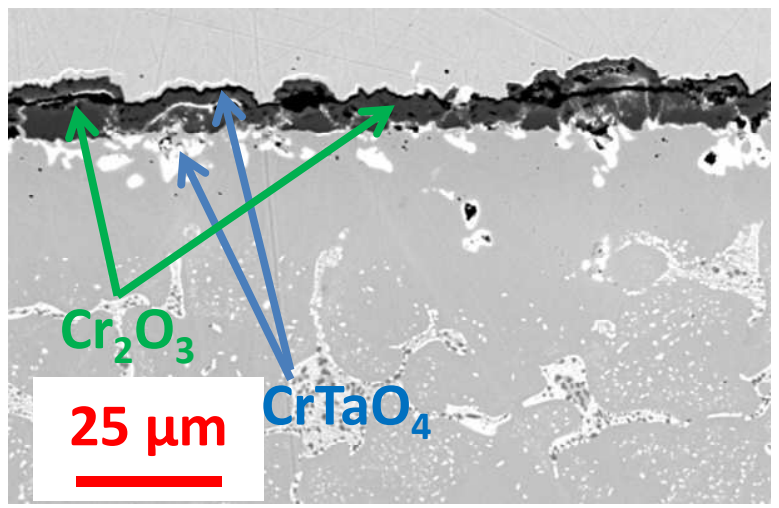

FIGURE 5. Cross-sectional examination of the oxide scales and subsurfaces of the two Ni-richest alloys (SEM/BSE; top: $\times 250$; bottom: $\times 1000)$. 


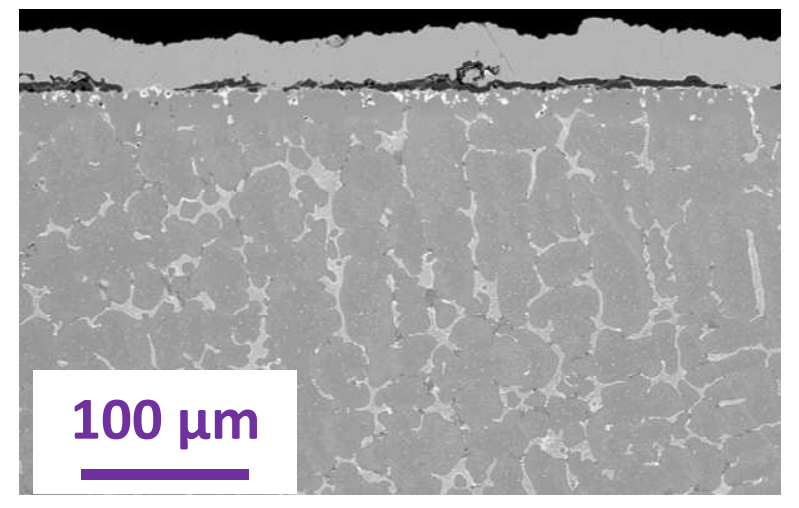

NiCo2 alloy $\left(24 \mathrm{~h} 1127^{\circ} \mathrm{C}\right)$
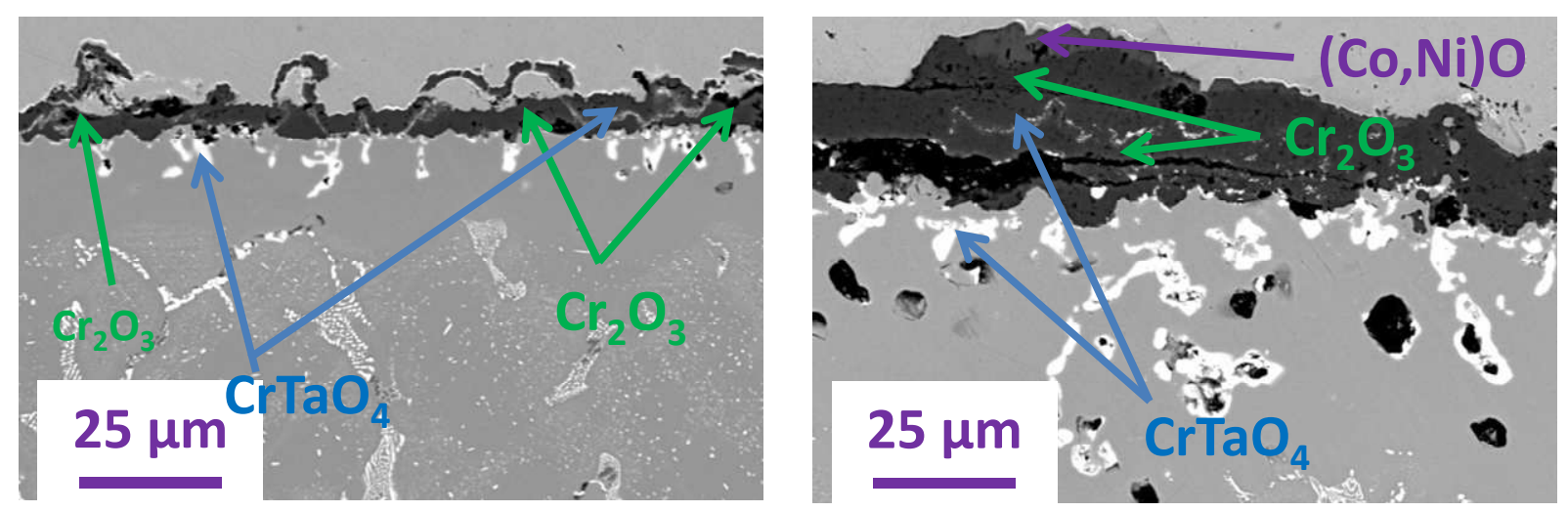

FIGURE 6. Cross-sectional examination of the oxide scales and subsurfaces of the two medium alloys (SEM/BSE; top: $\times 250$; bottom: $\times 1000)$. 


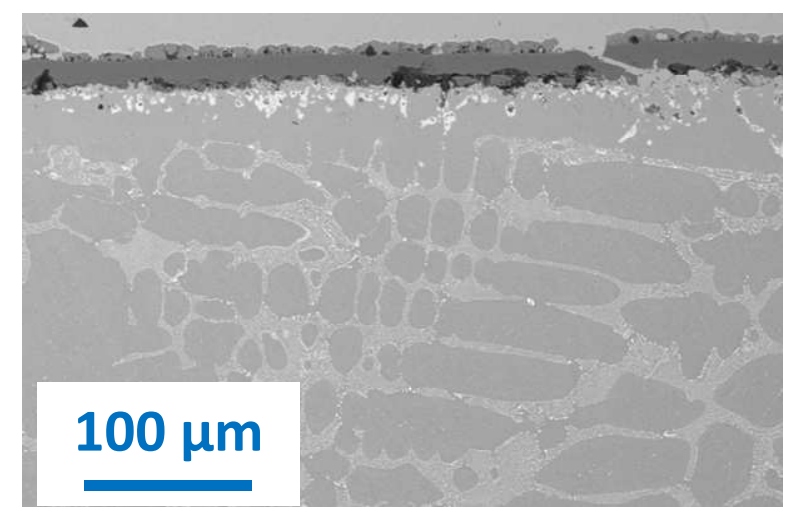

NiCo4 alloy $\left(24 \mathrm{~h} 1127^{\circ} \mathrm{C}\right)$

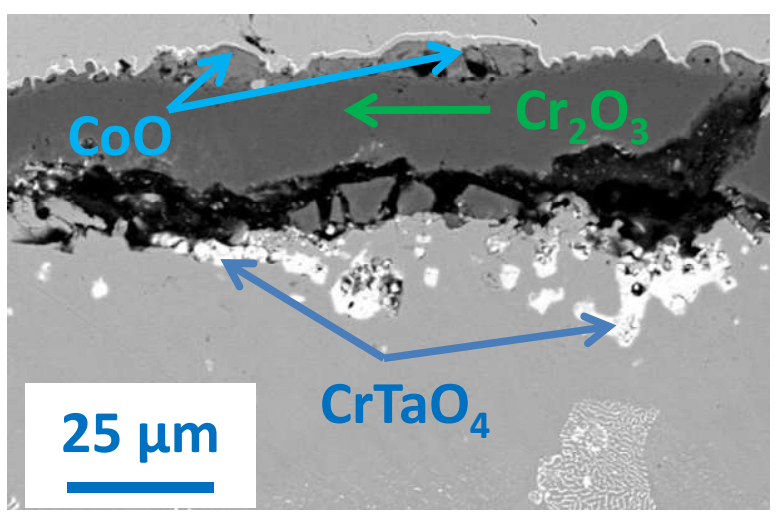

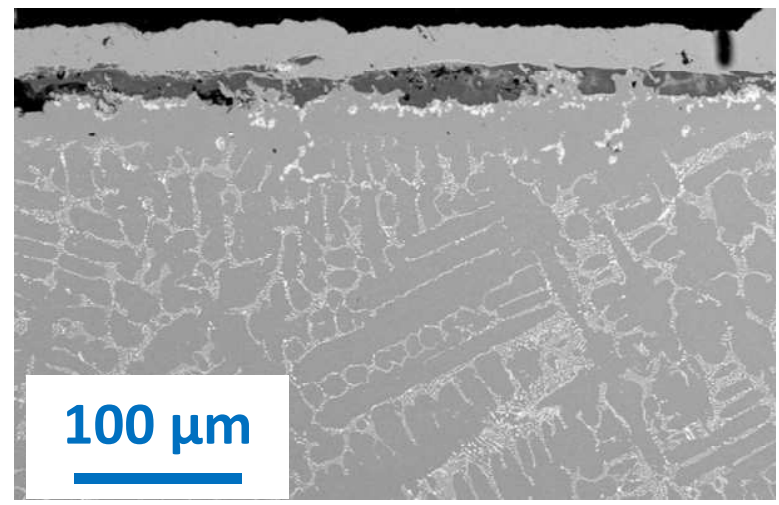

NiCo5 alloy $\left(24 \mathrm{~h} 1127^{\circ} \mathrm{C}\right)$

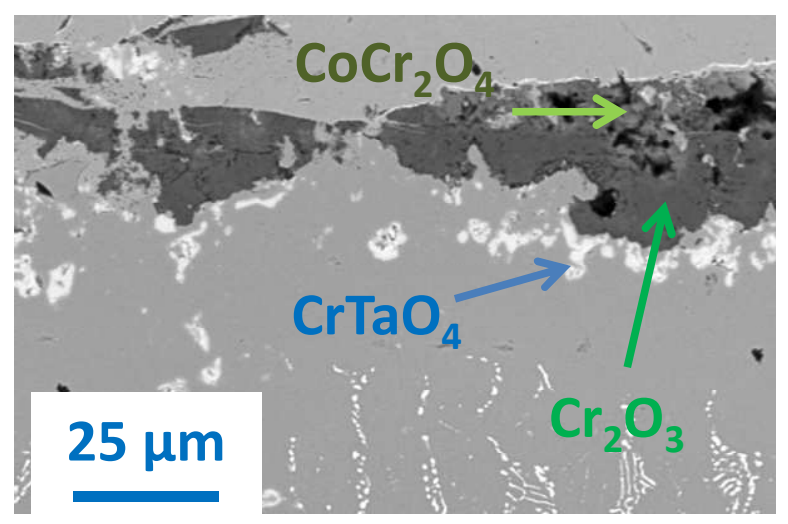

FIGURE 7. Cross-sectional examination of the oxide scales and subsurfaces of the two Co-richest alloys (SEM/BSE; top: $\times 250$; bottom: $\times 1000)$. 

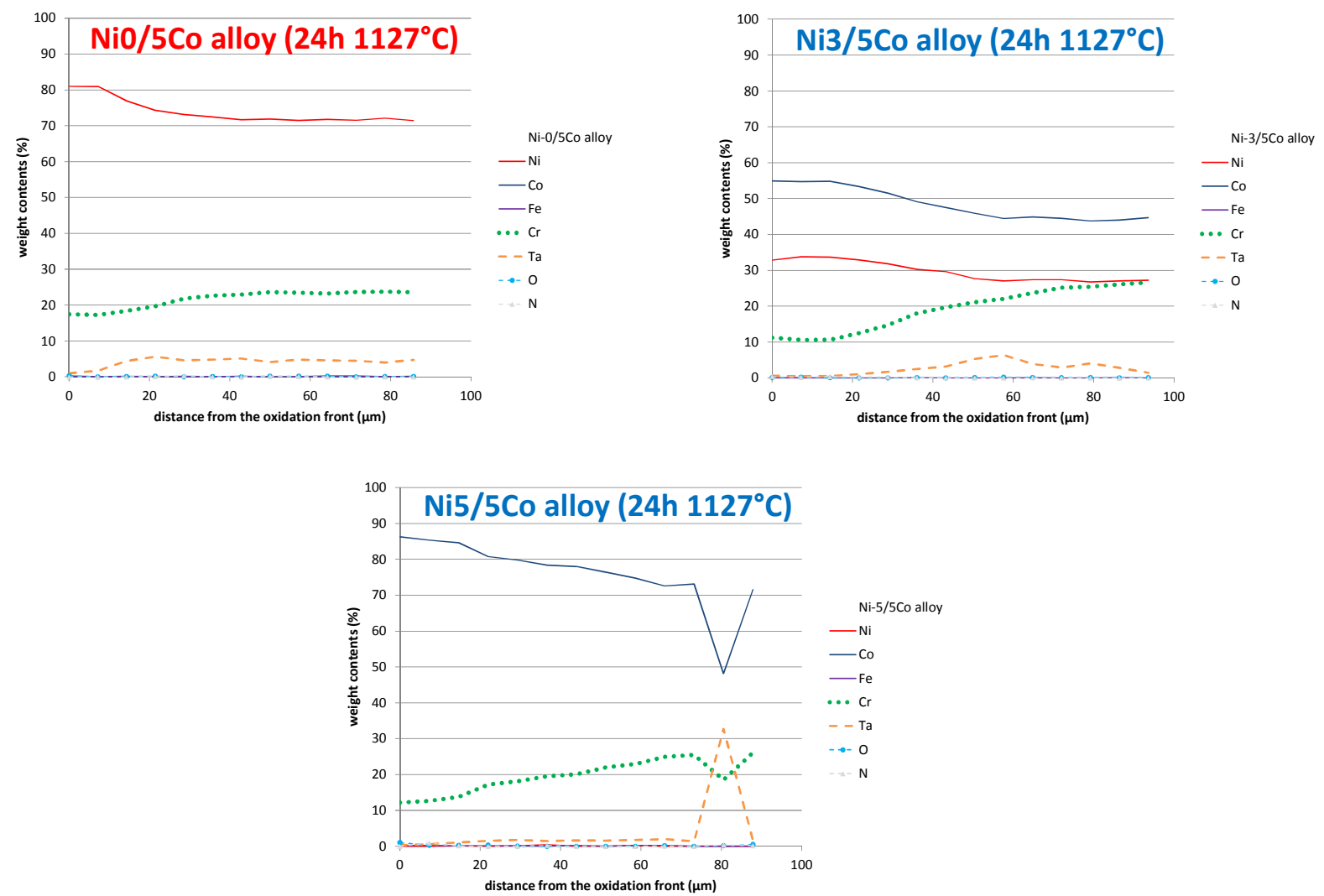

FIGURE 8. Concentration profiles in the subsurfaces of three of the six alloys (SEM/EDS); full scale. 

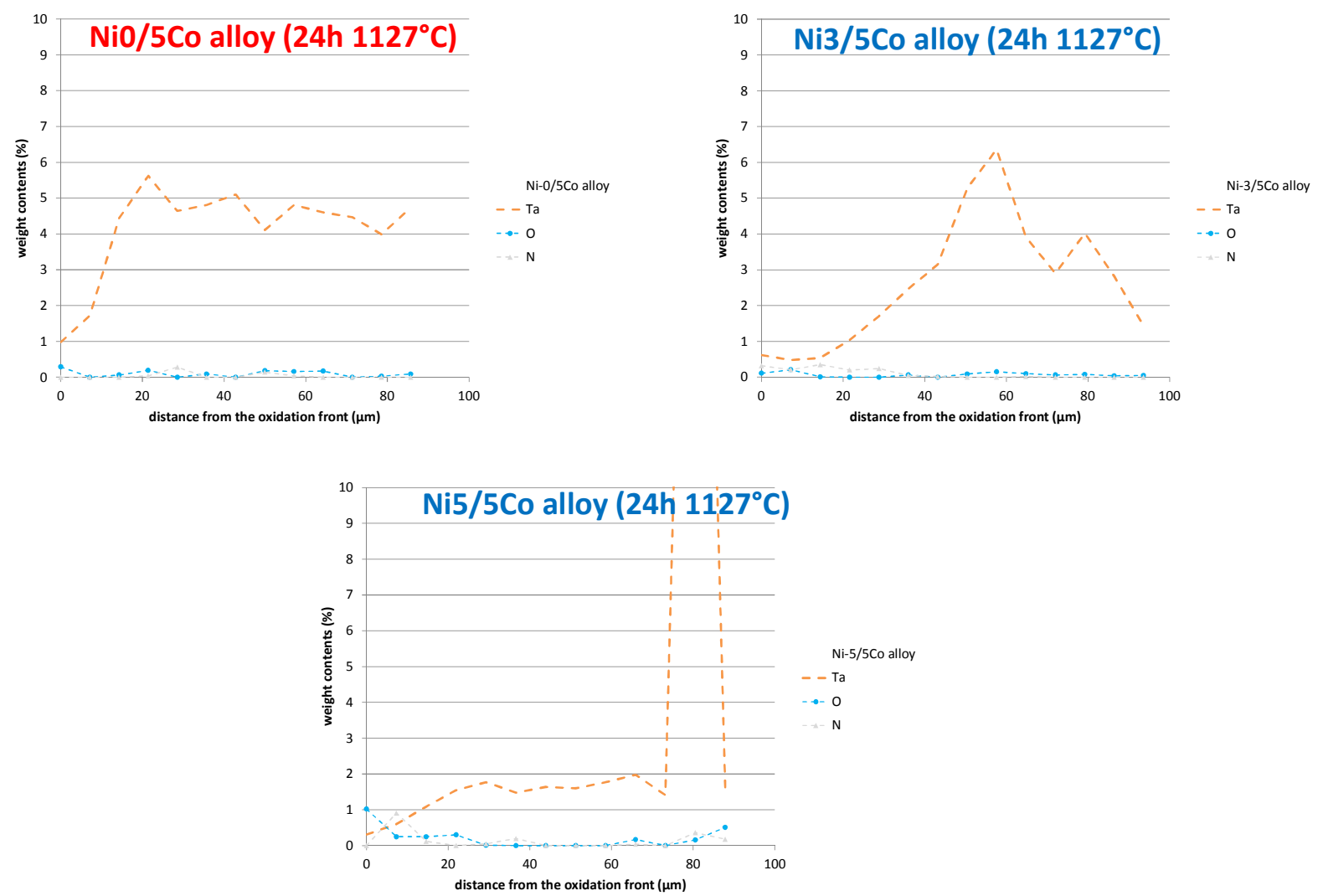

FIGURE 9. Concentration profiles in the subsurfaces of three of the six alloys (SEM/EDS); enlarged view. 


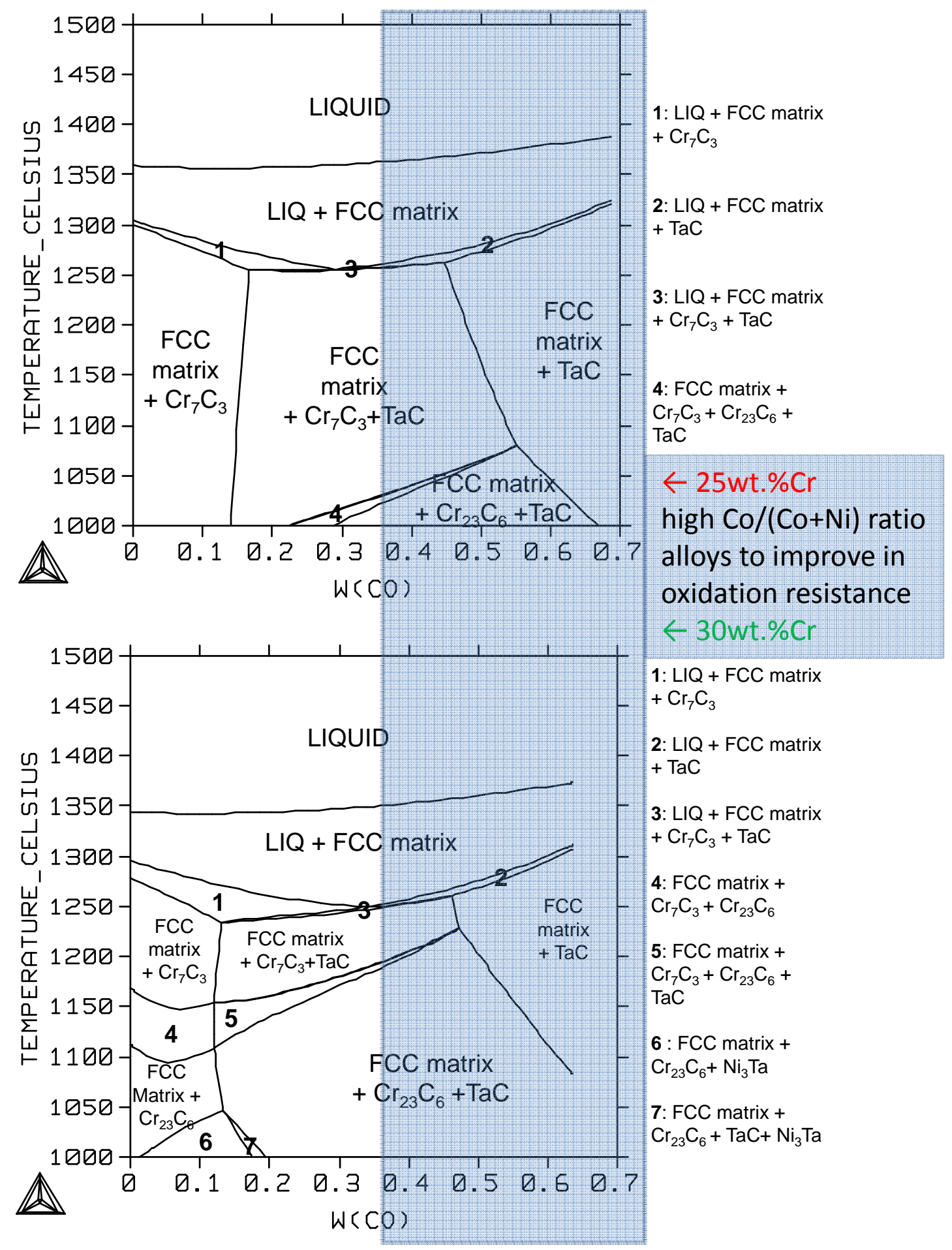

FIGURE 10. Effect of the addition of $5 \mathrm{wt} . \% \mathrm{Cr}$ on the $\{\mathrm{xCr}-0.4 \mathrm{C}-6 \mathrm{Ta}, \mathrm{wt} . \%\}$-isopleth section of the Ni-Co-Cr-TaC diagram (top: $25 \mathrm{wt} . \% \mathrm{Cr}$, bottom: 30wt.\%) 